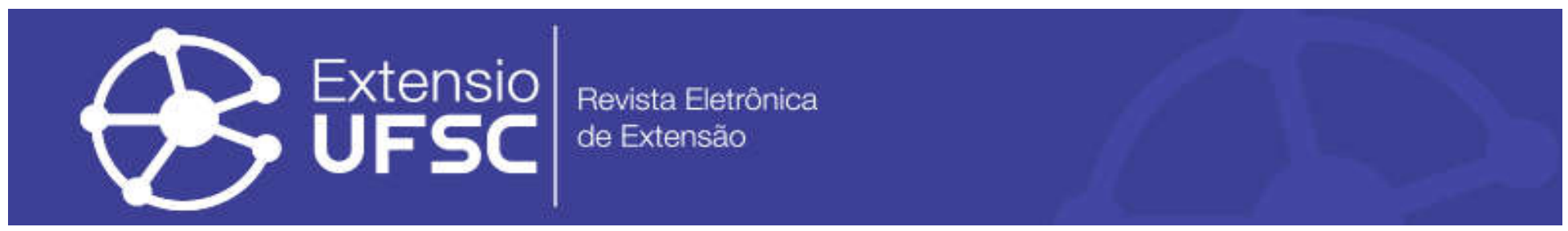

\title{
ORGANIZAÇÃO E REALIZAÇÃO DE UM GRUPO DE VIVÊNCIAS PARA PESSOAS EM PERÍODO PRÉ-OPERATÓRIO DE CIRURGIA PARA CONFECÇÃO DE ESTOMIA INTESTINAL: RELATO DE EXPERIÊNCIA
}

\author{
Eduarda Brito Dos Santos \\ Universidade Federal de Santa Catarina \\ eduardadsbrito@gmail.com \\ Lúcia Nazareth Amante \\ Universidade Federal de Santa Catarina \\ luciamante@gmail.com \\ Helena Sophia Strauss Mohr \\ Universidade Federal de Santa Catarina \\ helenaa.mohr@gmail.com
}

\author{
Maitê Mirian Will \\ Universidade Federal de Santa Catarina \\ maitewill2612@gmail.com \\ Andrelise Viana Rosa Tomasi \\ Universidade Federal de Santa Catarina \\ andrelisev@gmail.com \\ Maria Carolina Espindola \\ Universidade Federal de Santa Catarina \\ m.carol.esp@hotmail.com
}

Resumo

Objetivo: Relatar a experiência de organizar e realizar grupos de vivência para pessoas em período pré-operatório de cirurgia para confecção de estomia intestinal. Metodologia: Relato de experiência sobre a organização e realização de um grupo de vivências para os pacientes que estão em pré-operatório para confecção de estoma intestinal, que envolveu doze pacientes, oito familiares e os membros do grupo (uma professora, quatro estudantes da graduação em enfermagem, duas enfermeiras do hospital). Foi realizado de abril a dezembro de 2019, na unidade de internação cirúrgica do hospital universitário. Foram realizados quatro encontros. Resultados: compartilhamento de saberes populares e experiências entre os pacientes, familiares e facilitadores do encontro, acerca do processo de transição para a condição de vida com estomia intestinal, possibilitando adquirir conhecimentos a respeito desse novo processo de vida. Conclusão: Entende-se que a família, assim como o paciente, também vivencia esse processo de reabilitação e, portanto, necessita estar preparada para auxiliar nos cuidados.

Palavras-chave: Educação em Saúde. Estomia. Enfermagem Perioperatória.

\section{ORGANIZATION AND REALIZATION OF A GROUP OF EXPERIENCES FOR PEOPLE IN THE PREOPERATIVE PERIOD OF SURGERY FOR CONFECTION OF INTESTINAL OSTOMY: EXPERIENCE REPORT}

\begin{abstract}
Objective: To report the experience of organizing and conducting the living groups for people in the preoperative period of surgery for making an intestinal ostomy. Methodology: An experience report on the organization and conduct of a group of experiences for patients who are in pre- Operative procedure for making intestinal stoma, which involved 12 patients, eight family members and group members (one teacher, four undergraduate nursing students, two hospital nurses). It was carried out from April to December 2019, in the surgical hospitalization unit of the university hospital. Four meetings were held. Results: sharing of popular knowledge and experiences among patients, family members and facilitators of the meeting, about the transition process to the condition of life with an intestinal ostomy, making it possible to acquire knowledge about this new life process. Conclusion: It is understood that the family, as well as the patient, also experiences this rehabilitation process and, therefore, needs to be prepared to assist in care.

Keywords: Health Education. Stoma. Perioperative Nursing.
\end{abstract}


Organização e realização de um grupo de vivências para pessoas em período pré-operatório de cirurgia para confecção de estomia intestinal: relato de experiência

\section{ORGANIZACIÓN Y REALIZACIÓN DE UN GRUPO DE EXPERIENCIAS PARA PERSONAS EN EL PREOPERATORIO DE CIRUGÍA PARA CONFECCIÓN DE OSTOMÍA INTESTINAL: RELATO DE EXPERIENCIA}

Resumen

Objetivo: Informar la experiencia de organización y realización de los grupos de convivencia de personas en el período preoperatorio de cirugía para la preparación de ostomía intestinal Metodología: Informe de experiencia sobre la organización y realización de un grupo de experiencias para pacientes que se encuentran en procedimiento preoperatorio para la realización de estoma intestinal, que involucró a 12 pacientes, ocho familiares y miembros del grupo (un docente, cuatro estudiantes de licenciatura en enfermería, dos enfermeras del hospital). Se llevó a cabo de abril a diciembre de 2019 , en la unidad de hospitalización quirúrgica del hospital universitario. Se realizaron cuatro reuniones. Resultados: intercambio de conocimientos y experiencias populares entre pacientes, familiares y facilitadores del encuentro, sobre el proceso de transición a la condición de vida con ostomía intestinal, posibilitando adquirir conocimientos sobre este nuevo proceso de vida. Conclusión: Se entiende que la familia, así como el paciente, también vive este proceso de rehabilitación y, por tanto, necesita estar preparada para ayudar en la atención.

Palabras clave: Educación para la Salud. Estoma. Enfermería Perioperatoria. 
Organização e realização de um grupo de vivências para pessoas em período pré-operatório de cirurgia para confecção de estomia intestinal: relato de experiência

\section{INTRODUÇÃO}

Estomia consiste em uma abertura visceral através da pele, realizada por meio de ato cirúrgico, com a finalidade de promover respiração, alimentação ou eliminação. A estomia de eliminação intestinal é uma exteriorização de uma porção do intestino delgado ou grosso, indicada quando uma destas partes está impossibilitada, temporária ou permanentemente, de exercer suas funções. A finalidade é permitir a eliminação dos efluentes através da parede abdominal. Uma estomia de eliminação intestinal é caracterizada por colostomia (quando ocorre a exteriorização de uma porção do cólon, intestino grosso) e ileostomia (quando a exteriorização é de uma porção do íleo, intestino delgado) (INTERNATIONAL OSTOMY ASSOCIATION, 2017; BRASIL, 2009).

Doenças que acometem órgãos do sistema digestório, ou próximo dele, podem levar à confecção de estomia intestinal, especialmente as neoplasias de cólon e reto ou colorretal, as doenças intestinais e as diverticulares, além de causas externas, como: ferimento por arma branca, de fogo ou traumatismo contuso consequente a acidentes automobilísticos ou violência interpessoal (LEE et al., 2018; WOUND, OSTOMY AND CONTINENCE NURSES SOCIETY - WOCN, 2018).

Quando ocorre dano e a confecção da estomia é inevitável, a pessoa nessa situação enfrenta mudanças no contexto familiar, profissional, social e afetivo, o que ocasiona desequilíbrio na sua saúde, família e trabalho. A pessoa nutre sentimentos negativos ocasionados pela perda da autoestima e insatisfação com sua imagem corporal. Soma-se a isso ao desconhecimento acerca das políticas públicas voltadas ao usuário de estomia, o que acarreta déficit na busca por melhorias e de novas práticas de cuidado (MATOS et al., 2019; SILVA et al., 2017).

O enfermeiro atua com sua equipe em todo segmento hospitalar durante uma cirurgia de confecção de estomia. Desde o pré-operatório, período de medo, incerteza e fragilidade, quando são realizadas as orientações necessárias que buscam reduzir a ansiedade do paciente, bem como na demarcação do abdome; até o período pós-operatório com as instruções para as trocas dos equipamentos coletores e cuidados com a pele (MAURÍCIO et al., 2017; MARQUES et al., 2016).

Para que este cuidado ocorra de maneira eficiente, é necessário que os profissionais consigam compreender e atender as diferentes necessidades de cada pessoa com estomia intestinal, para que possam facilitar a recuperação e proporcionar o apoio suficiente no esclarecimento de dúvidas e superação de angústias em relação à estomia. Não basta que esses 
Organização e realização de um grupo de vivências para pessoas em período pré-operatório de cirurgia para confecção de estomia intestinal: relato de experiência

profissionais tenham conhecimento de novas tecnologias, mas é preciso pensar e propor um plano de cuidado individualizado fomentado de conhecimentos e didáticas para o ensinamento da utilização dos materiais necessários, a fim de promover o autocuidado (ARDIGO; AMANTE, 2013; MARQUES et al., 2016).

Sendo assim, a educação em saúde pode preparar a pessoa com estomia e sua família para enfrentar as dificuldades advindas desta condição, através da organização e implementação de ações de cuidado direcionadas às necessidades específicas dos envolvidos (DALMOLIN et al., 2020).

O Grupo de Apoio à Pessoa Ostomizada (GAO) foi criado em 1985, no Hospital Universitário da Universidade Federal de Santa Catarina, a partir do encontro de pacientes e profissionais da saúde e, desde então, vem promovendo a união de saberes acadêmicos e populares específicos de acordo com cada projeto proposto. Essas ações possuem baixo custo de implementação e alto potencial transformador, podendo muitas vezes oferecer soluções simples para problemas do cotidiano (SEIXAS, 2015).

A partir de reuniões periódicas deste grupo de extensão e encontros e orientações realizadas no Hospital Universitário Professor Polydoro Ernani de São Tiago, levantou-se a ideia de unir estas pessoas que possuem histórias tão diferentes, mas que agora se cruzam no ambiente hospitalar, para que elas possam compartilhar experiências e aprendizados acerca da estomia e oferecer apoio umas às outras.

Este estudo pretende, portanto, fundamentar-se no fato de que a confecção da estomia intestinal é um momento de transição impactante na vida das pessoas e seus familiares, pode ser compreendida como a possibilidade para a continuidade da vida. A pessoa deve ser incentivada ao processo de aceitação e de autocuidado pelos que irão passar ou que já passaram pelo procedimento cirúrgico.

Dessa forma, este trabalho objetiva relatar a experiência de organizar e realizar os grupos de vivência para pessoas em período pré-operatório de cirurgia para confecção de estomia intestinal.

\section{MATERIAIS E MÉTODOS}

Trata-se de um relato de experiência acerca de um grupo de vivências desenvolvido nas unidades de clínica cirúrgica de um hospital universitário de Florianópolis. As atividades tiveram início a partir do projeto de extensão "Ações em saúde e enfermagem para as pessoas com 
Organização e realização de um grupo de vivências para pessoas em período pré-operatório de cirurgia para confecção de estomia intestinal: relato de experiência

estomias intestinais: desenvolvimento de tecnologias para melhoria da qualidade de vida e inclusão social", aprovado pelo Sistema Integrado de Gerenciamento de Projetos de Pesquisa e de Extensão (SIGPEX) com o número 201818847.

Descrevem-se as distintas etapas do processo de idealizar, planejar e realizar o grupo de vivências. A idealização do grupo de vivências ocorreu nas reuniões do GAO, nas quais identificou-se uma lacuna na assistência prestada ao paciente em período perioperatório para a construção de uma estomia. O grupo de vivências foi idealizado por profissionais de saúde, estudantes de graduação e pós-graduação. Quanto ao planejamento, o grupo de vivências foi organizado em duas etapas: o acompanhamento dos pacientes e os encontros do grupo de vivências, que ocorreram paralelamente.

Participaram do grupo de vivências pacientes em período pré-operatório e pós-operatório de construção de estomia intestinal, internados e seus respectivos acompanhantes, sendo estes familiares ou pessoas do círculo social próximo, no total houve cerca de vinte participantes com média de quatro a cinco participantes por encontro. Pacientes que não possuíam indicação específica para a cirurgia de construção de estomia, mas que apresentavam possibilidade, também foram convidados a participar. Os encontros do grupo de vivências foram conduzidos por uma enfermeira e quatro acadêmicas de enfermagem que participaram do projeto.

A etapa "acompanhamento dos pacientes" consistiu na busca ativa por pacientes em perioperatório para a construção de estomia intestinal, internados nas Unidades de Internação Cirúrgica do Hospital Universitário. Por meio do contato com as enfermeiras das respectivas unidades de internação, as organizadoras identificavam os pacientes que preenchiam o critério de estar em período pré-operatório para confecção de estomia intestinal e passavam a ser acompanhados pela equipe do projeto de extensão. Nesta etapa, além do reconhecimento dos pacientes, realizava-se um contato primário visando aproximar os pacientes do grupo, convidando-os a participar dos encontros. Nesta oportunidade já se iniciavam as orientações sobre a confecção e cuidados com a estomia intestinal.

A frequência dos encontros aconteceu conforme a demanda no serviço de saúde, no total ocorreram quatro encontros, com duração de aproximadamente 1 hora cada, geralmente nas sextas feiras, às $11 \mathrm{~h}$, cada um com participantes diferentes e em torno de 12 pessoas por reunião, dentre eles pacientes, acompanhantes, professora e alunas. Os participantes variavam em cada encontro, porém todos os presentes participavam do início ao final. Este número variava devido aos períodos perioperatórios, pois como era semanal, um paciente que estava presente em um encontro na semana seguinte poderia estar em pós-operatório ou já ter recebido alta hospitalar. 
Organização e realização de um grupo de vivências para pessoas em período pré-operatório de cirurgia para confecção de estomia intestinal: relato de experiência

Os encontros do grupo de vivências são estruturados em quatro momentos, quais sejam: apresentação dos participantes; introdução à temática estomia; momento de discussão; e dinâmicas integrativas.

O primeiro momento do encontro do grupo de vivências consistiu na apresentação dos participantes. Os participantes e acompanhantes, depois de convidados pelas organizadoras do grupo, se dirigiam a uma sala de aula da unidade, reservada para a realização da atividade. Os participantes sentavam-se em semicírculo e eram estimulados a se apresentarem e compartilharem acerca da sua história.

No segundo momento, após a ambientação inicial com o grupo, realizava-se uma explicação sobre o Trato Gastrointestinal (TGI), a construção da estomia, os cuidados do período pós-operatório em relação aos cuidados com a estomia intestinal e sobre o retorno às atividades diárias.

No terceiro momento, realizava-se uma discussão acerca da temática apresentada, na qual os participantes expressavam impressões, dúvidas, sentimentos e expectativas relacionadas à cirurgia e à estomia.

O quarto momento consistia na realização de dinâmicas integrativas, que visavam aproximar o participante dos cuidados com a estomia, que facilitam e estimulam a familiarização com a condição de possuir estomia intestinal.

A etapa de "acompanhamento de pacientes" ocorreu ininterruptamente de abril a dezembro de 2019, sendo realizadas em média três visitas durante a semana. Neste período ocorreram quatro "encontros do grupo de vivências". Destaca-se que, no período de julho a setembro de 2019, houve poucas cirurgias para a construção de estomia intestinal, o que dificultou a realização dos encontros do grupo de vivência.

\section{RESULTADOS E ANÁLISES}

Parte-se do pressuposto, conforme Marques et al. (2016), de que o indivíduo que vivenciará a condição de possuir estomia intestinal necessita munir-se de novos conhecimentos que o ampare no desenvolvimento do seu autocuidado, bem como, permita uma nova rotina de vida com o mínimo de impactos e sofrimento, resultando na adaptação das atividades da vida diária. Nessa perspectiva, a atuação dos profissionais da equipe multidisciplinar vem ao encontro com as suas necessidades para promover a reabilitação e a melhora da qualidade de vida destes pacientes. 
Organização e realização de um grupo de vivências para pessoas em período pré-operatório de cirurgia para confecção de estomia intestinal: relato de experiência

Diante disso, destaca-se que, com a realização do grupo de vivências ao longo deste período, proporcionaram-se, como resultados principais, o compartilhamento de saberes populares e experiências entre os pacientes alvo da ação de extensão, seus acompanhantes e para os participantes que conduziram os encontros acerca do processo de transição para a condição de vida com estomia intestinal. Assim, possibilitou-se adquirir conhecimentos a respeito desse novo processo de vida. Tem-se que, com a integração dos pacientes com distintas histórias de vida, ainda que com a estomia como condição em comum, isso resultou em aprendizado mútuo que permeia desde a compreensão da cirurgia para confecção de uma estomia intestinal, até os cuidados no pós-operatório a serem desenvolvidos pelo paciente e/ou familiar.

Considera-se que, de acordo com Sousa et al. (2017), a vivência com estomia intestinal traz desafios e dificuldades para o indivíduo, acompanhados pela manifestação de dúvidas e medos que surgem desde o período pré-operatório e permanecem no pós-operatório. Esses sentimentos suscitados a partir da confecção de uma estomia intestinal dificultam o processo de uma vida saudável com estomia, à medida que essas incertezas e inseguranças o tornam frágeis, repercutindo de forma negativa na reabilitação do paciente. Nesse sentido, torna-se imprescindível que a pessoa com estomia compreenda a si mesmo e a realidade em que está inserida, para que assim possa exercer a sua autonomia no processo de autocuidado.

Com o grupo de vivências, obteve-se assim o estabelecimento de um contato prévio com a nova condição de vida, isto é, realizado antes da realização da cirurgia de confecção da estomia com o objetivo de reduzir e minimizar os impactos da estomia intestinal na vida da pessoa, nos aspectos físicos, psíquicos e sociais. Acrescenta-se que, por meio do diálogo realizado durante os encontros, criou-se uma abertura para a aproximação e familiaridade dos pacientes com a estomia, e isso resultou na compreensão do procedimento cirúrgico ao qual o paciente é submetido, bem como na projeção dos cuidados a serem desenvolvidos no período pósoperatório, possibilitando-se o reconhecimento de facilidades e dificuldades para com esse cuidado por parte do indivíduo e/ou familiar.

Com base em Ribeiro e Andrade (2020), são inúmeras as transformações que emergem no cotidiano do indivíduo a partir da construção de uma estomia intestinal, e aliado a isso, são diversos os questionamentos e inquietações a respeito desse novo processo, sendo indispensável a busca de recursos e estratégias de enfrentamento para conviver com a estomia intestinal, manter a autoestima e a qualidade de vida. Dessa forma, o profissional de enfermagem atua de modo a atender as necessidades dos pacientes com estomia intestinal, construir recursos para a reabilitação e autocuidado da pessoa com estoma intestinal, sendo a educação em saúde uma 
Organização e realização de um grupo de vivências para pessoas em período pré-operatório de cirurgia para confecção de estomia intestinal: relato de experiência

potente ferramenta. Os participantes receberam explicações sobre a anatomia e fisiologia do aparelho intestinal, sobre as causas e os locais para a confecção do estoma com a utilização de recursos visuais; explicação sobre os cuidados com o estoma. Foi oferecido o material utilizado para o cuidado dos estomas e estimulado o uso em moldes criados pelos próprios participantes.

Durante as atividades do grupo de vivências utilizamos algumas estratégias de educação em saúde, tais como exposição teórica sobre os conhecimentos acerca do funcionamento do intestino, de uma estomia e suas implicações e também utilizamos materiais como moldes de estomia.

Ainda, conforme Ribeiro e Andrade (2020), deve-se compreender a educação como um processo dinâmico, que é composto por elementos criativos, progressivos, reflexivos e libertadores, pautada no compartilhamento de saberes e aprendizados com a finalidade de destacar ao máximo as capacidades residuais e as potencialidades dos envolvidos no processo.

Nesse sentido, as atividades desenvolvidas no grupo de vivências possuíam exatamente esse caráter, de promover o aprendizado mútuo e extrair as potencialidades de cada um, facilitando dessa forma o reconhecimento de fragilidades e potencialidades para o processo de autocuidado desde o período pré-operatório, justamente com o objetivo de amenizar os sentimentos negativos que emergem durante este processo, e também os impactos de uma estomia intestinal.

As interações criadas a partir dos encontros resultaram na exposição de sentimentos e emoções dos pacientes e familiares, positivos e/ou negativos a respeito da estomia intestinal, tais como: ansiedade, medo, incerteza, insegurança e rejeição a respeito do novo processo de vida iminente. Nessa perspectiva, registramos a exteriorização dos sentimentos e emoções dos pacientes em face da construção da estomia intestinal, demandas dos profissionais e acadêmicos de enfermagem, a elaboração de estratégias e o fornecimento de subsídios para empoderar essas pessoas para o enfrentamento da nova condição e seu autocuidado, sendo este o mecanismo adotado para atender aos participantes dos grupos de vivências realizados no Hospital Universitário.

Aponta-se, em resumo, que o grupo de vivências favoreceu a compreensão do momento vivido pelos pacientes e familiares, o que certamente repercutirá positivamente no processo de viver de cada uma. Como limitação, idenficamos que a dinâmica do período perioperatório muitas vezes impede a participação dos pacientes. 
Organização e realização de um grupo de vivências para pessoas em período pré-operatório de cirurgia para confecção de estomia intestinal: relato de experiência

\section{CONSIDERAÇÕES FINAIS}

O grupo de vivências permitiu que observássemos, de uma forma mais profunda, o processo de aprendizagem dos pacientes e de seus familiares sobre as descobertas e desafios perioperatórios de um paciente que experiencia a construção de uma estomia intestinal. Escutamos, observamos e compreendemos suas dúvidas e preocupações e, em conjunto com o grupo, trocamos conhecimento teórico bem como as experiências de cada participante.

Salienta-se a relevância de se incluir a família desses pacientes no processo de reabilitação e aprendizado da pessoa com estomia. A família, por sua vez, também deve se sentir segura e confiante para apoiar e empoderar o paciente com estomia intestinal, principalmente em seu processo de autocuidados. Entende-se que a família, assim como o paciente, também vivencia esse processo de reabilitação e, portanto, necessita estar preparada para auxiliar nos cuidados necessários.

\section{REFERÊNCIAS}

ARDIGO, F. S.; AMANTE, L. N. Conhecimento profissional acerca do cuidado de enfermagem à pessoa com estomia intestinal e família. Texto Contex Enferm, Florianópolis, v. 22, n. 4, p. 1064-71, out./dez. 2013. Disponível em:

http://www.scielo.br/scielo.php?script=sci_arttext\&pid=S0104-07072013000400024. Acesso em: 13 ago. 2020.

BRASIL. Ministério da Saúde. Portaria n. ${ }^{\circ}$ 400, de 16 de novembro de 2009. Brasília, DF, 2009. Disponível em:

http://bvsms.saude.gov.br/bvs/saudelegis/sas/2009/prt0400_16_11_2009.html. Acesso em: 06 ago. 2020.

COMBER, H. et al. Causes and outcomes of emergency presentation of rectal cancer. Int J

Cancer, [s. 1], v. 139, n. 5, p. 1031-39, sep. 2016. Disponível em:

https://pubmed.ncbi.nlm.nih.gov/27087482/. Acesso em: 24 jul. 2020.

DALMOLIN, A. et al. Ações educativas de enfermagem às pessoas com estoma intestinal de eliminação: revisão narrativa. Saúde (Santa Maria), v. 46, n. 2, e43195, 2020. Disponível em: https://periodicos.ufsm.br/revistasaude/article/view/43195. Acesso em: 13 ago. 2020.

INTERNATIONAL OSTOMY ASSOCIATION. The stoma [internet] 2017. Disponível em: http://www.ostomyinternational.org/ostomy-help/stoma.html. Acesso em: 24 jul. 2020. 
Organização e realização de um grupo de vivências para pessoas em período pré-operatório de cirurgia para confecção de estomia intestinal: relato de experiência

LEE, T. H. et al. Aging, obesity, and the incidence of diverticulitis: a population-based study. Mayo Clin Proc, [s. 1], v. 93, n. 9, p. 1256-65, sep. 2018. Disponível em: https://www.ncbi.nlm.nih.gov/pmc/articles/PMC6200415/. Acesso em: 24 jul. 2020.

MARQUES, G. S. et al. A vivência de pessoas com estomia intestinal no grupo de apoio em um Hospital Universitário. Revista Hospital Universitário Pedro Ernesto, Rio de Janeiro, v. 15, n. 2, p. 113-21, abr./jun. 2016. Disponível em: https://www.epublicacoes.uerj.br/index.php/revistahupe/article/view/28235/0. Acesso em: 06 ago. 2020.

MARTINS, M. L. et al. A trajetória do grupo de apoio à pessoa ostomizada: projetando ações em saúde e compartilhando vivências e saberes. Texto Contex Enferm, Florianópolis, v. 14, n. 4, p. 594-600, out./dez. 2005. Disponível em: https://www.scielo.br/scielo.php?pid=S010407072005000400017\&script=sci_abstract\&tlng=pt. Acesso em: 06 ago. 2020.

MATOS, M. A. B. et al. As repercussões causadas pela incontinência urinária na qualidade de vida do idoso. Rev Fundam Care Online, [s. 1], v. 11, n. 3, p. 567-75, abr./jun. 2019. Disponível em: http://www.seer.unirio.br/index.php/cuidadofundamental/article/view/6581/pdf_1. Acesso em: 19 ago. 2020.

MAURÍCIO, V. C. et al. A visão dos enfermeiros sobre as práticas educativas direcionadas as pessoas estomizadas. Esc Anna Nery, Rio de Janeiro, v. 21, n. 4, e20170003, set. 2017. Disponível em: https://www.scielo.br/scielo.php?pid=S1414$81452017000400225 \&$ script=sci_arttext\&tlng=pt. Acesso em: 06 ago. 2020.

RIBEIRO, W. A.; ANDRADE, M. Perspectiva do paciente estomizado intestinal frente a implementação do autocuidado. Revista Pró-UniverSUS, [s. 1], v. 11, n. 1, p. 6-13, jan./jun. 2020. Disponível em: https://editora.universidadedevassouras.edu.br/index.php/RPU/article/view/2214. Acesso em: 13 ago. 2020.

SEIXAS, A. S. et al. As tecnologias sociais como instrumento para o desenvolvimento nacional. Revista GEINTEC, São Cristovão, v. 5, n. 4, p. 2678-88, 2015. Disponível em: http://www.revistageintec.net/index.php/revista/article/view/872/622. Acesso em: 06 ago. 2020 .

SILVA, N. M. et al. Psychological aspects of patients with intestinal stoma: integrative review. Rev Latino-Am. Enfermagem, [s. 1], v. 25, e2950, 2017. Disponível em: http://www.scielo.br/scielo.php?script=sci_arttext\&pid=S0104-11692017000100608. Acesso em: 13 ago. 2020.

SOUSA, A. R. A. et al. Estratégias educativas para pessoas com estomia intestinal: revisão integrativa. Revista Enfermagem Atual, [s. 1], v. 81, n. 19, p. 81-8, 2017. Disponível em: https://revistaenfermagematual.com/index.php/revista/article/view/325. Acesso em: 13 ago. 2020.

UNIVERSIDADE FEDERAL DE SANTA CATARINA. Pró Reitoria de Extensão. Catálogo de Extensão. Florianópolis: PROEX/UFSC, 2018. p. 243. 
Organização e realização de um grupo de vivências para pessoas em período pré-operatório de cirurgia para confecção de estomia intestinal: relato de experiência

WOUND, OSTOMY AND CONTINENCE NURSES SOCIETY - WOCN. Guideline developmentt ask force. WOCN Society clinical guideline: management of the adult patient with a fecal or urinary ostomy - na executive summary. J Wound Ostomy Continence Nurs, [s. 1], v. 45, Issue 1, p. 50-8, jan./ feb. 2018. Disponível em:

https://journals.lww.com/jwocnonline/Fulltext/2018/01000/WOCN_Society_Clinical_Guideli ne_Management_of_the.9.aspx. Acesso em: 09 set. 2020.

Recebido em: 15/09/2020

Aceito em: 15/04/2021 